ARTICLE

Received 28 Jan 2014 | Accepted 30 Apr 2014 | Published 28 May $2014 \quad$ DOl: 10.1038/ncomms5021

\title{
Near room-temperature multiferroic materials with tunable ferromagnetic and electrical properties
}

Hong Jian Zhao ${ }^{1,2,3}$, Wei Ren ${ }^{4}$, Yurong Yang ${ }^{2,3}$, Jorge Íñiguez ${ }^{5}$, Xiang Ming Chen ${ }^{1} \&$ L. Bellaiche ${ }^{2,3}$

The quest for multiferroic materials with ferroelectric and ferromagnetic properties at room temperature continues to be fuelled by the promise of novel devices. Moreover, being able to tune the electrical polarization and the paramagnetic-to-ferromagnetic transition temperature constitutes another current research direction of fundamental and technological importance. Here we report on the first-principles-based prediction of a specific class of materialsnamely, $\mathrm{R}_{2} \mathrm{NiMnO}_{6} / \mathrm{La}_{2} \mathrm{NiMnO}_{6}$ superlattices where $\mathrm{R}$ is a rare-earth ion-that exhibit an electrical polarization and strong ferromagnetic order near room temperature, and whose electrical and ferromagnetic properties can be tuned by means of chemical pressure and/or epitaxial strain. Analysis of the first-principles results naturally explains the origins of these highly desired features.

\footnotetext{
${ }^{1}$ Laboratory of Dielectric Materials, Department of Materials Science and Engineering, Zhejiang University, Hangzhou 310027, China. ${ }^{2}$ Institute for Nanoscience and Engineering, University of Arkansas, Fayetteville, Arkansas 72701, USA. ${ }^{3}$ Department of Physics, University of Arkansas, Fayetteville, Arkansas 72701, USA. ${ }^{4}$ Department of Physics, Shanghai University, 99 Shangda Road, Shanghai 200444, China. ${ }^{5}$ Institut de Ciència de Materials de Barcelona (ICMAB-CSIC), Campus UAB, 08193 Bellaterra, Spain. Correspondence and requests for materials should be addressed to X.M.C. (email: xmchen59@zju.edu.cn) or to L.B. (email: laurent@uark.edu).
} 
T he search for multiferroic materials combining electric and magnetic behaviours in a single phase has attracted a lot of attention from the perspective of designing new devices ${ }^{1-7}$ and deepening our knowledge of materials and electromagnetism. One particularly important current research direction aims at the discovery of materials that possess electrical polarization and are ferromagnetic at room temperature. Strikingly, it appears that these two desired features rarely coexist ${ }^{4,6,8,9}$. For instance, $\mathrm{Bi}_{2} \mathrm{MnReO}_{6}$ and $\mathrm{Bi}_{2} \mathrm{NiReO}_{6}$ have been predicted to exhibit an electrical polarization at room temperature, but both the Re and $\mathrm{Ni} / \mathrm{Mn}$ sublattices adopt an antiferromagnetic order, therefore yielding a 'ferrimagnetic' rather than ferromagnetic overall ordering near $300 \mathrm{~K}$ (ref. 4). Similarly, ferrimagnetism (or weak magnetization) instead of ferromagnetism occurs in other compounds possessing a room-temperature electrical polarization, and that is the case for $\mathrm{GaFeO}_{3}$ (ref. 6), hexagonal $\mathrm{LuFeO}_{3}$ epitaxial thin films ${ }^{7}$ and the solid solutions formed by mixing lead zirconium titanate and lead iron tantalite perovskites ${ }^{10}$. In other words, systems exhibiting both a significant electrical polarization and a strong magnetization near room temperature are very scarce (as also consistent with refs 11,12).

On the other hand, double perovskite $\mathrm{Bi}_{2} \mathrm{NiMnO}_{6}$ has been recently synthesized and found to be ferroelectric below $485 \mathrm{~K}$ and ferromagnetic below $140 \mathrm{~K}$ (ref. 13). While this latter ordering occurs below room temperature, and therefore makes $\mathrm{Bi}_{2} \mathrm{NiMnO}_{6}$ useless for practical applications, Azuma et al. ${ }^{13}$ have been critical in pointing out the possibility of investigating double perovskites for the search of ferromagnets having a polarization near $300 \mathrm{~K}$. Double perovskite oxides $\mathrm{R}_{2} \mathrm{NiMnO}_{6}$ (where $\mathrm{R}$ is a rare-earth element) ${ }^{14}$ are thus attractive, especially since $\mathrm{La}_{2} \mathrm{NiMnO}_{6}$ has a ferromagnetic Curie temperature $T_{\mathrm{cm}} \sim 287 \mathrm{~K}$ and $T_{\mathrm{cm}}$ is nearly linearly dependent on (and thus tunable with) the radius of the rare-earth ion in $\mathrm{R}_{2} \mathrm{NiMnO}_{6}$ (ref. 14). Unfortunately, one shortcoming of the $\mathrm{R}_{2} \mathrm{NiMnO}_{6}$ systems is that they are 'not' ferroelectric but rather possess antipolar displacements resulting ${ }^{15}$ from their oxygen octahedral tilting pattern $\left(a^{-} a^{-} c^{+}\right.$in Glazer's notation $\left.{ }^{66}\right)$. Nevertheless, the possibility of inducing a spontaneous electrical polarization at room temperature in $\mathrm{R}_{2} \mathrm{NiMnO}_{6}$-based double perovskite oxides may exist, for example, by mixing them with $\mathrm{La}_{2} \mathrm{NiMnO}_{6}$ compounds to form short-period (001)-oriented $\mathrm{R}_{2} \mathrm{NiMnO}_{6} /$ $\mathrm{La}_{2} \mathrm{NiMnO}_{6}$ superlattices. As a matter of fact, recent studies ${ }^{2,17}$ have shown that (001)-oriented $\left(\mathrm{ABO}_{3}\right)_{1} /\left(\mathrm{A}^{\prime} \mathrm{BO}_{3}\right)_{1}$ superlattices can exhibit an electrical polarization if both $\mathrm{ABO}_{3}$ and $\mathrm{A}^{\prime} \mathrm{BO}_{3}$ materials adopt an $a^{-} a^{-} c^{+}$tilting pattern, because of the mechanism called hybrid improper ferroelectricity ${ }^{17}$. It is therefore legitimate to wonder whether (001) $\mathrm{R}_{2} \mathrm{NiMnO}_{6} /$ $\mathrm{La}_{2} \mathrm{NiMnO}_{6}$ superlattices can be ferromagnetic and have an electrical polarization near room temperature.

Here we report that such features can indeed be realized in these systems. Moreover, we show that their electrical polarization and magnetic Curie temperature can be tuned (and thus adjusted to desired values) by varying the chemical pressure (that is, the size of the rare-earth ion) and/or the epitaxial strain imposed by the substrates on which the $\mathrm{R}_{2} \mathrm{NiMnO}_{6} / \mathrm{La}_{2} \mathrm{NiMnO}_{6}$ superlattice films can be grown. Finally, this study also allows us to better understand the mechanism of hybrid improper ferroelectricity, by providing the atomistic energy and interactions that are responsible for it in $\mathrm{R}_{2} \mathrm{NiMnO}_{6} / \mathrm{La}_{2} \mathrm{NiMnO}_{6}$ systems.

\section{Results}

Structure and magnetic ordering in $\mathrm{La}_{2} \mathrm{NiMnO}_{6}$. As detailed in the Methods section, first-principles calculations are performed on $\mathrm{La}_{2} \mathrm{NiMnO}_{6}$ systems and on $\mathrm{R}_{2} \mathrm{NiMnO}_{6} / \mathrm{La}_{2} \mathrm{NiMnO}_{6}$ superlattices for which the $\mathrm{Ni}$ atoms only have $\mathrm{Mn}$ atoms as firstnearest neighbours of the B-sublattice and vice versa.

Let us first compare our predicted lattice parameters and magnetic moments of bulk $\mathrm{La}_{2} \mathrm{NiMnO}_{6}$, in its $\mathrm{P}_{1} / n$ ground state, to experimental data, in order to check the accuracy of our approach. Ferromagnetic order of $\mathrm{Ni}$ and $\mathrm{Mn}$ ions is assumed here. The $\mathbf{a}, \mathbf{b}$ and $\mathbf{c}$ lattice vectors in the 20 -atom $\mathrm{P} 2{ }_{1} / n$ unit cell of $\mathrm{La}_{2} \mathrm{NiMnO}_{6}$ are along the pseudo-cubic [110], [-110] and [001] directions, respectively, and their magnitudes are calculated to be 5.466, 5.425 and $7.676 \AA$, respectively. They therefore agree rather well (namely, within $0.73 \%$ ) with the results measured at $3.5 \mathrm{~K}$ (ref. 18), which are 5.506, 5.456 and $7.732 \AA$, respectively. Our computed magnetic moments for $\mathrm{Ni}^{2+}$ and $\mathrm{Mn}^{4+}$ are 1.54 and $3.13 \mu_{\mathrm{B}}$, respectively. This is in reasonable agreement with both experimental $\left(1.9 \mu_{\mathrm{B}} \text { for } \mathrm{Ni}^{2+} \text { and } 3.0 \mu_{\mathrm{B}} \text { for } \mathrm{Mn}^{4+}\right)^{18}$ and previous first-principles $\left(1.43 \mu_{\mathrm{B}}\right.$ for $\mathrm{Ni}^{2}{ }^{+}$and $3.0 \mu_{\mathrm{B}}$ for $\left.\mathrm{Mn}^{4+}\right)^{19}$ results.

Ferroelectric ordering in the superlattices. We initially build the $\mathrm{R}_{2} \mathrm{NiMnO}_{6} / \mathrm{La}_{2} \mathrm{NiMnO}_{6}$ superlattices by substituting $\mathrm{La}^{3+}$ ions by $\mathrm{R}^{3+}$ ions in every other AO (001) plane of the perovskite structure, and then relax both atomic positions and lattice vectors. Let us do such substitution by starting from the relaxed $\mathrm{P} 2{ }_{1} /$ $n$ ground state of $\mathrm{La}_{2} \mathrm{NiMnO}_{6}$ (still assuming ferromagnetic order) and replacing the second $\mathrm{LaO}$ layer of our 20 -atom periodic supercell by RO layers (see Fig. 1a). While the $\mathrm{R}_{2} \mathrm{NiMnO}_{6}$ and $\mathrm{La}_{2} \mathrm{NiMnO}_{6}$ bulk compounds exhibit a paraelectric $\mathrm{P}_{1} / n$ ground state, the $\mathrm{R}_{2} \mathrm{NiMnO}_{6} / \mathrm{La}_{2} \mathrm{NiMnO}_{6}$ superlattices are all found to adopt an electrical polarization (and a polar $\mathrm{P}_{1}$ space group) after the full structural relaxation is reached. This electrical polarization is found here to be antiparallel to the in-plane $\mathbf{b}$ axis. Interestingly, we also numerically found that it can lie parallel to that axis when the $\mathrm{R}$ ions occupy the first, rather than second, $\mathrm{AO}$ layer of the 20-atom cell of our $\mathrm{R}_{2} \mathrm{NiMnO}_{6} /$ $\mathrm{La}_{2} \mathrm{NiMnO}_{6}$ superlattices. Figure $2 \mathrm{a}$ further shows that the magnitude of this polarization increases on decreasing the rareearth ionic radius, $r_{\mathrm{R}}$, therefore revealing the possibility of continuously tuning of this polarization via the so-called chemical pressure associated with the different size of the rare-earth ions ${ }^{20,21}$. In fact, since the ionic radius of $\mathrm{La}$ in $\mathrm{La}_{2} \mathrm{NiMnO}_{6}$ or $\mathrm{R}_{2} \mathrm{NiMnO}_{6} / \mathrm{La}_{2} \mathrm{NiMnO}_{6}$ material is about $1.16 \AA$ (ref. 22; La has a coordination number of eight in these compounds), Fig. 2a also shows that the in-plane electrical polarization is enhanced when the difference of ionic radius between $\mathrm{R}$ and $\mathrm{La}$ increases. Practically, the electrical polarization ranges between $1.4 \mu \mathrm{C} \mathrm{cm}{ }^{-2}$ for the $\mathrm{Ce}_{2} \mathrm{NiMnO}_{6} / \mathrm{La}_{2} \mathrm{NiMnO}_{6}$ system and $9.2 \mu \mathrm{Ccm}^{-2}$ for the $\mathrm{Er}_{2} \mathrm{NiMnO}_{6} / \mathrm{La}_{2} \mathrm{NiMnO}_{6}$ superlattice, such values being large enough to be experimentally detected. They are also of the same order as the in-plane electrical polarization predicted to exist, via the so-called hybrid improper ferroelectricity, in the $\mathrm{BiFeO}_{3} / \mathrm{LaFeO}_{3}$ superlattice grown on a (001)-oriented $\mathrm{SrTiO}_{3}$ substrate ${ }^{2}$ and in some other $\left(\mathrm{ABO}_{3}\right)_{1} /$ $\left(\mathrm{A}^{\prime} \mathrm{BO}_{3}\right)_{1}$ superlattices ${ }^{17}$ combining two pure $\mathrm{ABO}_{3}$ and $\mathrm{A}^{\prime} \mathrm{BO}_{3}$ materials that adopt the $a^{-} a^{-} c^{+}$tilting pattern and are paraelectric. (Note that $a$ and $c$ in this notation of the tilting pattern refer to rotations around the pseudo-cubic $<001>$ axes.)

Interestingly, our superlattices also possess a similar tilting pattern, with antiphase tilting of the $\mathrm{NiO}_{6}$ and $\mathrm{MnO}_{6}$ octahedra in the $(\mathrm{a}, \mathrm{b})$ plane as well as in-phase tilting about the out-of-plane c direction. Let us therefore determine whether the mechanism of hybrid improper ferroelectricity, that technically relies on an energy of the form $\lambda \mathrm{Q}_{1} \mathrm{Q}_{2} \mathrm{P}$ based on symmetry arguments (where $\lambda$ is a constant, $Q_{1}$ and $Q_{2}$ are in-plane antiphase and outof-plane in-phase tilting modes, respectively, and $\mathrm{P}$ is the in-plane 
a

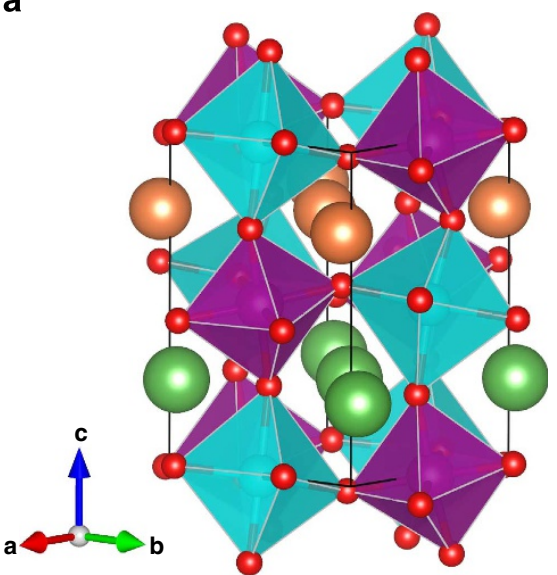

b

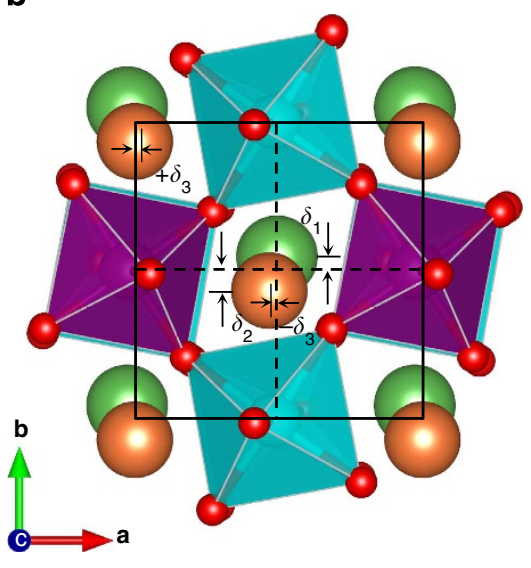

Figure 1 | $\mathbf{P 2} \mathbf{1}_{\mathbf{1}}$ ground state of the studied $\mathbf{R}_{\mathbf{2}} \mathbf{N i M n \mathbf { O } _ { 6 }} / \mathbf{L} \mathbf{a}_{\mathbf{2}} \mathbf{N i M n \mathbf { M } _ { \mathbf { 6 } }}$ superlattices. (a) Representation in three dimensions. (b) Representation in the (a,b) plane. The $\mathrm{La}^{3+}, \mathrm{R}^{3+}, \mathrm{Ni}^{2+}, \mathrm{Mn}^{4+}$ and $\mathrm{O}^{2-}$ ions are displayed as green, orange, cyan, purple and red spheres, respectively. $\mathrm{The}^{\mathrm{NiO}} 6$ and $\mathrm{MnO}_{6}$ octahedra are displayed using cyan and purple octahedra, respectively. The largest magnitude of the antipolar displacement $\left(\delta_{2}\right)$ of the $\mathrm{R}^{3+}$ ions with respect to those $\left(\delta_{1}\right)$ of the $\mathrm{La}^{3+}$ ions along the $\mathbf{b}$ axis is schematized here to emphasize that this inhomogeneity is the microscopic reason for the creation of the electrical polarization. The antipolar displacements along the $\mathbf{a}$ axis of the R ions within the RO plane (denoted as $+\delta_{3}$ and $-\delta_{3}$ ) are also shown. As examples, $\delta_{1}, \delta_{2}$ and $\delta_{3}$ are equal to $0.048,0.070$ and 0.017 , respectively, in lattice constant units in $\mathrm{Er}_{2} \mathrm{NiMnO}_{6} / \mathrm{La}_{2} \mathrm{NiMnO}_{6}$ (and $0.024,0.027$ and 0.006 , respectively, in $\mathrm{Ce}_{2} \mathrm{NiMnO}_{6} / \mathrm{La}_{2} \mathrm{NiMnO}_{6}$ ).

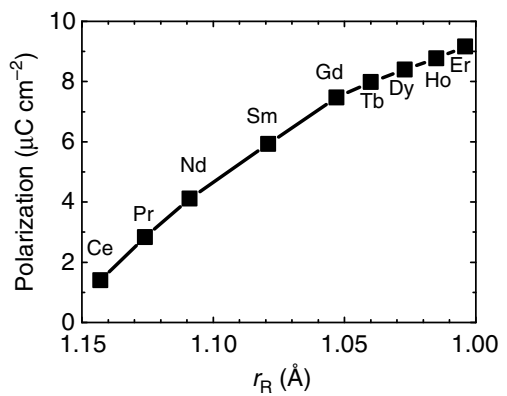

C

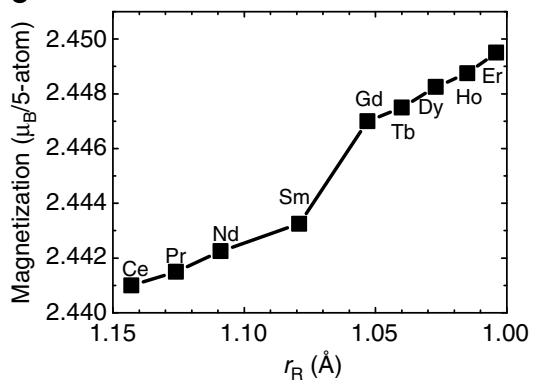

b

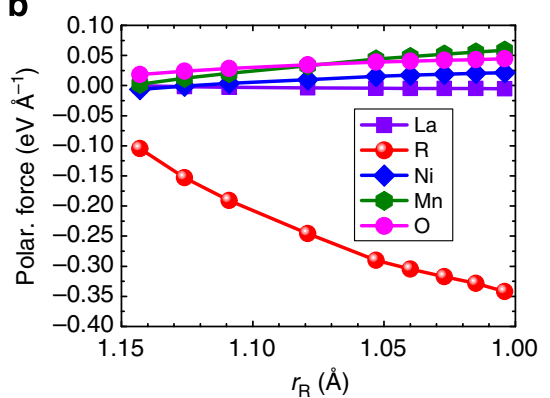

d

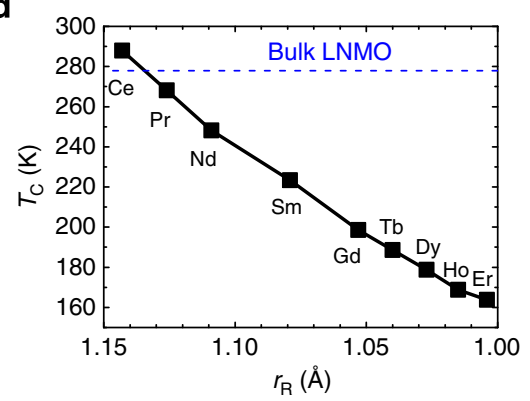

Figure 2 | Properties of $\mathbf{R}_{\mathbf{2}} \mathbf{N i M n O}_{6} / \mathbf{L a}_{\mathbf{2}} \mathbf{N i M n O}_{\mathbf{6}}$ systems as a function of the rare-earth ionic radius. (a,c,d) The predicted electric polarization (as computed from Berry phase), magnetization (at $T=0 \mathrm{~K}$ ) and paramagnetic-to-ferromagnetic transition temperature (as calculated by Monte Carlo simulation) in the relaxed $\mathrm{P} 2{ }_{1}$ ground state of the superlattices, respectively. (b) The average forces (along the $\mathbf{b}$ direction) on $\mathrm{La}^{3+}, \mathrm{R}^{3+}, \mathrm{Ni}^{2+}, \mathrm{Mn}^{4+}$ and $\mathrm{O}^{2-}$ ions in the unrelaxed state of the $\mathrm{R}_{2} \mathrm{NiMnO}_{6} / \mathrm{La}_{2} \mathrm{NiMnO}_{6}$ superlattice, for which the lattice parameters and atomic coordinates are those of the paraelectric $\mathrm{P}_{1} / n$ state of $\mathrm{La}_{2} \mathrm{NiMnO}_{6}$ and for which the $\mathrm{R}$ ions occupy the second $\mathrm{AO}$ layer of Fig. 1a. The blue horizontal dashed line in $\mathbf{d}$ represents the magnetic Curie temperature of $\mathrm{La}_{2} \mathrm{NiMnO}_{6}$ bulk. As evidenced in Supplementary Fig. 2, the error bar of the paramagnetic-to-ferromagnetic transition temperature can be estimated to be about $6 \mathrm{~K}$. Moreover, an error bar of polarization can be estimated by comparing the results of the relaxed structure of the $\mathrm{Er}_{2} \mathrm{NiMnO}_{6} / \mathrm{La}_{2} \mathrm{NiMnO}_{6}$ superlattice when using $U=3 \mathrm{eV}$ and $U=5 \mathrm{eV}$. It is found to be as small as $1 \%$ (which corresponds to a variation of the polarization from 9.16 to $9.26 \mu \mathrm{C} \mathrm{cm}^{-2}$ ).

polarization), also applies to our investigated systems. If it does apply, we also wish to provide a microscopic understanding for the formation of the electrical polarization in our systems. For that, it is important to first recall that Bellaiche and Íniguez ${ }^{15}$ demonstrated, via the introduction of specific atomistic interactions giving rise to the energy that was denoted $\Delta E_{1}$, that any $a^{-} a^{-} c^{+}$tilting pattern in any perovskite material should also generate antipolar motions of the A atoms that are parallel to the $\mathbf{b}$ direction in one (001) AO plane and then antiparallel to the $\mathbf{b}$ axis in the next (001) AO plane. Such 
antipolar displacements of the La ions are indeed numerically found in the relaxed $\mathrm{P}_{1} / n$ state of bulk $\mathrm{La}_{2} \mathrm{NiMnO}_{6}$. We then decided to start from this latter state of $\mathrm{La}_{2} \mathrm{NiMnO}_{6}$ (adopting ferromagnetism), then substitute the second $\mathrm{LaO}$ layer by an $\mathrm{RO}$ layer (in our periodic 20-atom supercell) to create the $\mathrm{R}_{2} \mathrm{NiMnO}_{6} / \mathrm{La}_{2} \mathrm{NiMnO}_{6}$ superlattice shown in Fig. 1a, but preventing any ionic relaxation from occurring after such substitution is done. The resulting average forces along the $\mathbf{b}$ direction for all types of ions are displayed in Fig. $2 \mathrm{~b}$ as a function of the rare-earth ionic radius ${ }^{22}$. Note that there are no average forces on any kind of atom along the a and $\mathbf{c}$ axes and, by average force, we mean the sum of the forces on the same kind of atoms divided by the number of these forces. Three important features are revealed by this figure. First of all, the force on the $\mathrm{R}^{3+}$ ions is the dominant one among all forces felt by ions along the $\mathbf{b}$ direction. Second, this force on the $\mathrm{R}^{3+}$ ions is antiparallel to the b axis (interestingly, we further found that this force is parallel to the $\mathbf{b}$ direction if the $\mathrm{R}$ ions occupy the first AO layer). Third, the magnitude of this force increases as $r_{\mathrm{R}}$ decreases. It is thus clear that the electrical polarization of the fully relaxed $\mathrm{R}_{2} \mathrm{NiMnO}_{6} /$ $\mathrm{La}_{2} \mathrm{NiMnO}_{6}$ superlattices shown in Fig. 2a mostly originates from the fact that the opposite antipolar motions (along the $\mathbf{b}$ direction) of $\mathrm{R}$ and $\mathrm{La}$ ions do not have the same magnitude, as schematized in Fig. $1 \mathrm{~b}$ and also consistent with refs 2,23. Such an inhomogeneity of antipolar motions can be understood when realizing that the $\Delta E_{1}$ atomistic energy provided in ref. 15 naturally leads (because of the $a^{-} a^{-} c^{+}$tilting pattern) to the (001) LaO layers of Fig. 1 experiencing a 'global, macroscopiclike' energy of the form $-\mathrm{K}_{1, \mathrm{La}} \omega_{\mathrm{R}} \omega_{\mathrm{M}} \mathrm{u}_{\mathrm{La}}$ where $\mathrm{K}_{1, \mathrm{La}}$ is a positive constant, $\omega_{R}$ and $\omega_{M}$ are scalars characterizing the in-plane antiphase and out-of-plane in-phase tiltings, respectively ${ }^{24}$, and $\mathrm{u}_{\mathrm{La}}$ is the displacement of the La ions along the $\mathbf{b}$ axis with respect to their ideal positions. Similarly, the (001) RO layers of Fig. 1 have an associated energy of the form $+K_{1, R} \omega_{R} \omega_{M} u_{R}$, where $K_{1, R}$ is another positive constant (which is different from $\mathrm{K}_{1, \mathrm{La}}$ because of the different chemical nature of the AO layers) and $u_{R}$ is the displacement of the $\mathrm{R}$ ions along the $\mathbf{b}$ direction. By summing these two different energies and introducing the polarization $\mathrm{P}=\left(\mathrm{u}_{\mathrm{La}}+\mathrm{u}_{\mathrm{R}}\right) / 2$ and an antiferroelectric vector $\mathrm{A}=\left(\mathrm{u}_{\mathrm{La}}-\mathrm{u}_{\mathrm{R}}\right) / 2$ (associated with the X-point of the five-atom cubic Brillouin zone), one obtains a total energy that can be decomposed into two novel parts, namely $-\left(\mathrm{K}_{1, \mathrm{La}}+\mathrm{K}_{1, \mathrm{R}}\right) \omega_{\mathrm{R}} \omega_{\mathrm{M}} \mathrm{A}$ and $-\left(K_{1, L a}-K_{1, R}\right) \omega_{R} \omega_{M} P$. The former is responsible for the existence of a homogenous in-plane antiferroelectric vector in the superlattice, while the latter precisely characterizes the socalled hybrid improper ferroelectricity ${ }^{17,23}$. Note that the effective charges of $\mathrm{La}$ and $\mathrm{R}$ ions are found to be rather similar along the b axis (namely, around 4.15), with their differences typically ranging between $2 \%$ (when $\mathrm{R}=\mathrm{Ce}$ ) and $7 \%$ (when $\mathrm{R}=\mathrm{Er}$ ). One can thus directly relate polarization with ionic displacements of $\mathrm{La}$ and $\mathrm{R}$ ions, in first approximation (we numerically found that the difference in effective charges between La and R ions typically results in a change of a polarization that is five times smaller than the polarization arising from the assumption that $\mathrm{La}$ and $\mathrm{R}$ ions have the same average effective charge in the $\mathrm{R}_{2} \mathrm{NiMnO}_{6} /$ $\mathrm{La}_{2} \mathrm{NiMnO}_{6}$ superlattices). Note also that the fact that Fig. 2a shows that the magnitude of the polarization is enhanced when the difference in size between the $\mathrm{R}$ and La ions increases implies that the coefficient in front of the second aforementioned energy, namely $\mathrm{K}_{1, \mathrm{La}}-\mathrm{K}_{1, \mathrm{R}}$, becomes larger as the size difference between $\mathrm{R}$ and $\mathrm{La}$ ions is bigger. Interestingly, if we start again from the symmetry-invariant energy $\Delta E_{1}$ provided in ref. 15 and consider the same tilting pattern as above (that is, same values of $\omega_{R}$ and $\left.\omega_{\mathrm{M}}\right)$, but choose the alternate ordering of the (001) $\mathrm{LaO}$ and $\mathrm{RO}$ planes (that is, with respect to the example above, we exchange the locations of $\mathrm{La}$ and $\mathrm{R}$ atoms), one can show that the polarization-inducing energy term turns out to be $-\left(\mathrm{K}_{1, \mathrm{R}}-\mathrm{K}_{1, \mathrm{La}}\right) \omega_{\mathrm{R}} \omega_{\mathrm{M}} \mathrm{P}$, that is, the same as above but with opposite sign. The minimization of this energy, via structural relaxation of the $\mathrm{A}$ atoms, implies that the polarization will then switch its direction when the $\mathrm{R}$ ions occupy the first (rather than the second) AO layer of the used 20-atom periodic supercell. Note that, ultimately, the phase matching between the La/R ordering and the antiphase rotations $\omega_{\mathrm{R}}$ determines the polarization direction. Note also that, and as demonstrated in refs 2,25 , the polarization of systems possessing trilinear coupling energy (as it is the case here) is switchable, which also typically leads to a switch of oxygen octahedral tiltings. It will also be interesting to determine in future studies whether this polarization switching is homogenous or (non)inhomogenous in nature (especially when defects are present in the grown sample). Finally, let us point out that, as shown in Fig. 1b, any two first-nearest neighbouring $\mathrm{R}$ (respectively, La) ions in any (001) AO plane move in an antiparallel manner to each other along the a axis-that is, along the in-plane direction that is perpendicular to the polarization. Such an additional displacement originates from the $\Delta E_{2}$ atomistic energy of ref. 15, which induces another antiferroelectric vector (in this case associated with the R-point of the five-atom cubic Brillouin zone) as a result of the $a^{-} a^{-} c^{+}$tilting pattern. Such additional displacements average out to zero in any (001) AO plane, and therefore do not give rise to any component of the macroscopic spontaneous polarization along the a axis.

Magnetic ordering in the superlattices. Having understood the origin of the polarization in the $\mathrm{R}_{2} \mathrm{NiMnO}_{6} / \mathrm{La}_{2} \mathrm{NiMnO}_{6}$ superlattices, it is clear that this polarization should remain non-zero at room temperature since the energy $-\left(\mathrm{K}_{1, \mathrm{La}}-\mathrm{K}_{1, \mathrm{R}}\right) \omega_{\mathrm{R}} \omega_{\mathrm{M}} \mathrm{P}$ implies that the polarization is driven by the oxygen octahedral tiltings and both the antiphase and in-phase oxygen octahedral tiltings are known to exist at ambient conditions in both $\mathrm{La}_{2} \mathrm{NiMnO}_{6}$ and $\mathrm{R}_{2} \mathrm{NiMnO}_{6}$ pure materials ${ }^{26}$. Let us now check whether these superlattices can also exhibit a significant magnetization at $300 \mathrm{~K}$, which would thus make them roomtemperature multiferroics. First, note that Fig. 2c shows that the computed magnetization of the $\mathrm{R}_{2} \mathrm{NiMnO}_{6} / \mathrm{La}_{2} \mathrm{NiMnO}_{6}$ superlattices at $T=0 \mathrm{~K}$ is large (of the order of $2.4-2.5 \mathrm{Bohr}$ magneton per five atoms) for any $\mathrm{R}$ ion, as consistent with a strong ferromagnetism. To determine whether this magnetization can persist up to room temperature, we calculate the paramagnetic-to-ferromagnetic Curie point of $\mathrm{R}_{2} \mathrm{NiMnO}_{6} /$ $\mathrm{La}_{2} \mathrm{NiMnO}_{6}$ superlattices (see Fig. 2d). We first investigate the Curie temperature of bulk $\mathrm{R}_{2} \mathrm{NiMnO}_{6}$ and $\mathrm{La}_{2} \mathrm{NiMnO}_{6}$ double perovskites as a function of the rare-earth ionic radius, arising from the use of different Hubbard $U$ values (see Supplementary Fig. 1 and Supplementary Discussion). The calculated Curie temperatures are systematically overestimated compared with the experimental values ${ }^{14}$. In addition, the increasing of Hubbard $U$ enhances $T_{C}$ in bulk $\mathrm{R}_{2} \mathrm{NiMnO}_{6}$ and $\mathrm{La}_{2} \mathrm{NiMnO}_{6}$. Thus, we multiplied the first-principles-derived exchange constants (with Hubbard $U 3.0 \mathrm{eV}$ for both $\mathrm{Ni}$ and $\mathrm{Mn}$ ) of all the spin Hamiltonians used in this work by a factor of 0.685 , which is the correction needed so that the Heisenberg Hamiltonians reproduce all the experimental paramagnetic-to-ferromagnetic transition temperatures of the different $\mathrm{R}_{2} \mathrm{NiMnO}_{6}$ systems ${ }^{14}$ (see Supplementary Fig. 2 and Supplementary Discussion). The calculated Curie temperature of $\mathrm{R}_{2} \mathrm{NiMnO}_{6} / \mathrm{La}_{2} \mathrm{NiMnO}_{6}$ superlattices decreases from $\sim 290$ to $\sim 165 \mathrm{~K}$ when going from $\mathrm{R}=\mathrm{Ce}$ to $\mathrm{R}=\mathrm{Er}$. As a result, the magnetic temperature of $\mathrm{R}_{2} \mathrm{NiMnO}_{6} / \mathrm{La}_{2} \mathrm{NiMnO}_{6}$ is significantly tunable (that is, by $>100 \mathrm{~K})$ via the chemical pressure associated with the size of the $\mathrm{R}$ ions, and $\mathrm{Ce}_{2} \mathrm{NiMnO}_{6} / \mathrm{La}_{2} \mathrm{NiMnO}_{6}$ superlattice is a 
specifically promising candidate for designing a near roomtemperature multiferroic material. These two findings are of obvious technological importance. Please note that our calculated Curie temperature of $\mathrm{Ce}_{2} \mathrm{NiMnO}_{6} / \mathrm{La}_{2} \mathrm{NiMnO}_{6}$ superlattice is the largest one compared with the other eight $\mathrm{R}_{2} \mathrm{NiMnO}_{6} /$ $\mathrm{La}_{2} \mathrm{NiMnO}_{6}$ superlattices as well as bulk $\mathrm{La}_{2} \mathrm{NiMnO}_{6}$. This leads us to gain more insight into $\mathrm{La}_{2} \mathrm{NiMnO}_{6}$ and $\mathrm{R}_{2} \mathrm{NiMnO}_{6}$. Thus we investigate the correlation between Curie temperature of bulk $\mathrm{R}_{2} \mathrm{NiMnO}_{6}$ perovskites and antiphase as well as in-phase tilting angles (see Supplementary Figs 3 and 4 and Supplementary Discussion).

Effects of epitaxial strain on physical properties. All the aforementioned calculations concern fully relaxed $\mathrm{R}_{2} \mathrm{NiMnO}_{6} /$ $\mathrm{La}_{2} \mathrm{NiMnO}_{6}$ systems. However, in some cases, some lattice vectors are imposed by external conditions and thus cannot relax to their equilibrium values. This is the case for epitaxial (001) thin films, for which the in-plane lattice parameters are those of the substrate on top of which the films are grown. It is thus relevant to investigate the effect of an epitaxial misfit strain on properties of the $\mathrm{R}_{2} \mathrm{NiMnO}_{6} / \mathrm{La}_{2} \mathrm{NiMnO}_{6}$ superlattices. For that purpose, the calculated electric polarization and Curie temperature of (001)oriented films made of $\mathrm{Ce}_{2} \mathrm{NiMnO}_{6} / \mathrm{La}_{2} \mathrm{NiMnO}_{6}, \mathrm{Pr}_{2} \mathrm{NiMnO}_{6} /$ $\mathrm{La}_{2} \mathrm{NiMnO}_{6}, \mathrm{Sm}_{2} \mathrm{NiMnO}_{6} / \mathrm{La}_{2} \mathrm{NiMnO}_{6}, \mathrm{Er}_{2} \mathrm{NiMnO}_{6} / \mathrm{La}_{2} \mathrm{NiMnO}_{6}$ and $\mathrm{La}_{2} \mathrm{NiMnO}_{6}$, are shown in Fig. 3a,b as a function of the inplane lattice parameter $\left(\mathrm{a}_{\mathrm{IP}}\right)$. Our calculated results first show that the electric polarization of the investigated $\mathrm{R}_{2} \mathrm{NiMnO}_{6} /$ $\mathrm{La}_{2} \mathrm{NiMnO}_{6}$ superlattices is basically independent of the epitaxial strain, with the exception of $\mathrm{Er}_{2} \mathrm{NiMnO}_{6} / \mathrm{La}_{2} \mathrm{NiMnO}_{6}$ for which the polarization slightly increases from $\sim 9.0$ to $\sim 10.5 \mu \mathrm{C} \mathrm{cm}^{-2}$ when $\mathrm{a}_{\mathrm{IP}}$ decreases from 3.96 to $3.68 \AA$. This insensitivity of the polarization with strain is technologically useful since it implies that the polarization persists for a large choice of available substrates. It is also fundamentally interesting since it goes against the common knowledge that in-plane polarization of (001) films should rapidly decrease, and even disappear, when decreasing the in-plane lattice constant (that is, when the strain becomes compressive and large in magnitude $)^{27-29}$. In fact, and as consistent with what was recently found for rare-earth orthochromates ${ }^{20}$, such a unusual and weak dependence of the in-plane polarization on the strain is due to the fact that a larger epitaxial compression tends to reduce the in-plane antiphase tilting $\omega_{\mathrm{R}}$ while increasing the out-of-plane in-phase tilting $\omega_{\mathrm{M}}$. As a result, the product $\omega_{R} \omega_{M}$ is largely independent of the strain, and so is the in-plane polarization that depends on this $\omega_{R} \omega_{M}$ product via the aforementioned hybrid improper ferroelectricity mechanism.

Moreover, Fig. $3 \mathrm{~b}$ shows that the paramagnetic-to-ferromagnetic Curie temperature of the superlattices (and $\mathrm{La}_{2} \mathrm{NiMnO}_{6}$ ) is rather sensitive to the epitaxial strain, as consistent with ref. 30 and magnetostriction: as $\mathrm{a}_{\mathrm{IP}}$ decreases, the Curie temperature goes up significantly at first, to eventually become weakly $\mathrm{a}_{\mathrm{IP}}$ dependent. Figure $3 b$ therefore shows that one can also create a near room-temperature multiferroic, and tune magnetic transition temperature, by applying an appropriate epitaxial strain in the $\mathrm{R}_{2} \mathrm{NiMnO}_{6} / \mathrm{La}_{2} \mathrm{NiMnO}_{6}$ superlattices. In particular, (001) $\mathrm{Ce}_{2} \mathrm{NiMnO}_{6} / \mathrm{La}_{2} \mathrm{NiMnO}_{6}$ films are promising materials to exhibit magnetization and polarization above $290 \mathrm{~K}$, when grown on any substrate having an in-plane lattice constant ranging between 3.70 and $3.80 \AA$ (which corresponds to an average compressive epitaxial strain experienced by $\mathrm{Ce}_{2} \mathrm{NiMnO}_{6} / \mathrm{La}_{2} \mathrm{NiMnO}_{6}$ films varying between $-3.9 \%$ and $-1.3 \%$ ).

\section{Discussion}

We have discovered that (and understood why) (001)-oriented $\mathrm{R}_{2} \mathrm{NiMnO}_{6} / \mathrm{La}_{2} \mathrm{NiMnO}_{6}$ superlattices can adopt an electrical

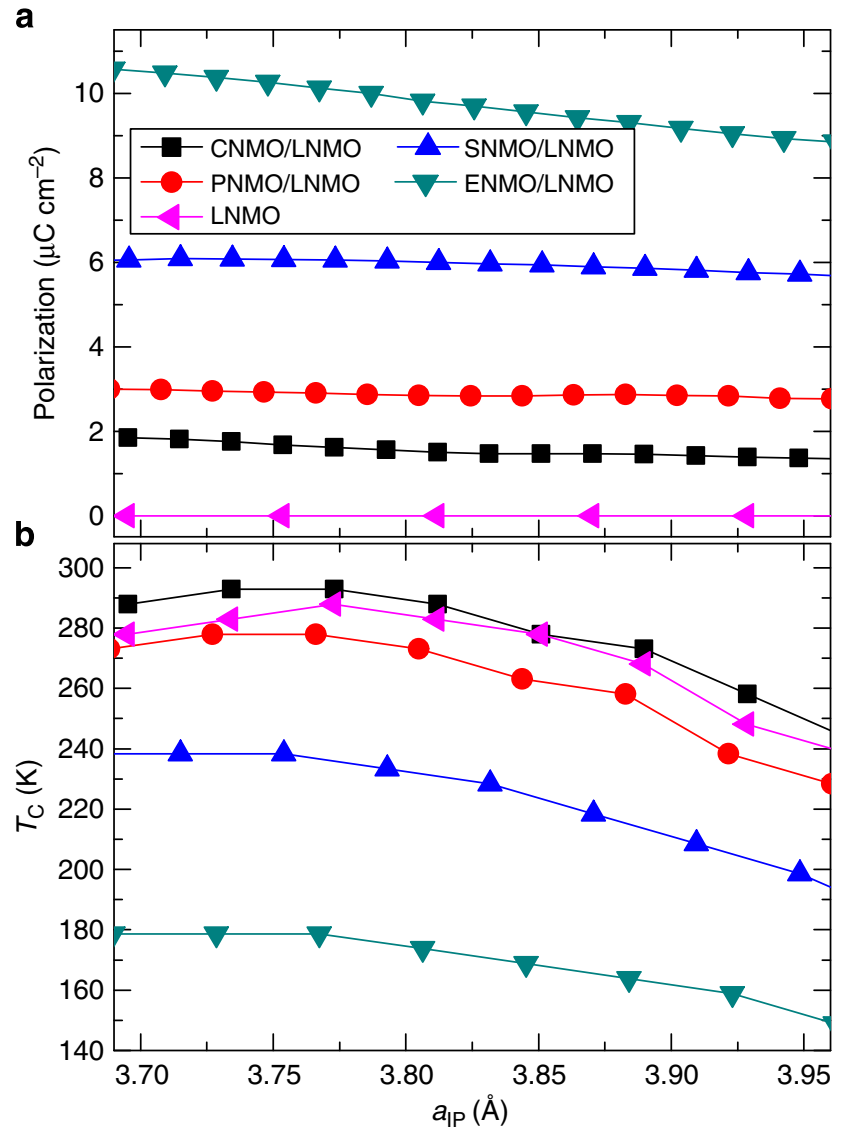

Figure 3 | Properties of epitaxial (001) films made of several superlattices as a function of the in-plane lattice parameter. These superlattices are $\mathrm{Ce}_{2} \mathrm{NiMnO}_{6} / \mathrm{La}_{2} \mathrm{NiMnO}_{6}(\mathrm{CNMO} / \mathrm{LNMO}), \mathrm{Pr}_{2} \mathrm{NiMnO}_{6} /$ $\mathrm{La}_{2} \mathrm{NiMnO}_{6}(\mathrm{PNMO} / \mathrm{LNMO}), \mathrm{Sm}_{2} \mathrm{NiMnO}_{6} / \mathrm{La}_{2} \mathrm{NiMnO}_{6}$ (SNMO/LNMO), $\mathrm{Er}_{2} \mathrm{NiMnO}_{6} / \mathrm{La}_{2} \mathrm{NiMnO}_{6}(\mathrm{ENMO} / \mathrm{LNMO})$ and $\mathrm{La}_{2} \mathrm{NiMnO}_{6}(\mathrm{LNMO})$, as a function of the in-plane lattice parameter. (a) The electric polarization as computed from Berry phase. (b) The magnetic Curie temperature as calculated by Monte Carlo simulation. As indicated in the captions of Fig. 2, the error bar for the polarization is estimated to be of the order of $1 \%$ while the error bar of the paramagnetic-to-ferromagnetic transition temperature is estimated to be about $6 \mathrm{~K}$.

polarization and be ferromagnetic essentially up to room temperature. Moreover, our first-principles calculations also reveal that the electric and magnetic properties can be tuned by chemical pressure and/or epitaxial strain in these systems. We thus hope that our predictions will soon be experimentally confirmed and used to design novel spintronic devices. Note that these predictions are for $\mathrm{R}_{2} \mathrm{NiMnO}_{6} / \mathrm{La}_{2} \mathrm{NiMnO}_{6}$ superlattice systems that are perfectly chemically ordered, and that disordering on the A- or B-sites likely will affect physical properties. It is thus important for experimentalists to fully control atomic ordering in the grown samples in order to be able to check our predictions. We are optimistic that our proposed $\mathrm{R}_{2} \mathrm{NiMnO}_{6} /$ $\mathrm{La}_{2} \mathrm{NiMnO}_{6}$ superlattices will be successfully grown in a near future based on the facts that similar compounds (namely, $\mathrm{NaRMnWO}_{6}$ and, especially, $\mathrm{Bi}_{2} \mathrm{NiMnO}_{6} / \mathrm{La}_{2} \mathrm{NiMnO}_{6}$ superlattices) have already been synthesized ${ }^{31,32}$. Note also that polarization and magnetism are coupled in our $\mathrm{R}_{2} \mathrm{NiMnO}_{6} /$ $\mathrm{La}_{2} \mathrm{NiMnO}_{6}$ superlattices since polarization is typically found to be enhanced by around $3 \%$ when going from the ferromagnetic to ferrimagnetic state (for which $\mathrm{Mn}$ and $\mathrm{Ni}$ ions being nearest neighbours to each other have opposite direction of their 
magnetic moment as well as different magnitudes for these moments). It is also important to realize that not all possible devices using multiferroics require a strong coupling between polarization $(\mathrm{P})$ and magnetization $(\mathrm{M})$. For instance, one can think of a four-state memory for which $+/-\mathrm{P}$ and $+/-\mathrm{M}$ can be accessed by using electric and magnetic fields, respectively, and for which it is desired to have a weak (rather than strong) coupling between $\mathrm{P}$ and $\mathrm{M}^{33}$. We also hope that other roomtemperature ferromagnetic multiferroics will be discovered following the general design principles employed here-namely: to combine two paraelectric compounds that are both ferromagnetic within a superlattice, create an electrical polarization in the resulting superlattice via the so-called hybrid improper ferroelectricity mechanism, and tune electrical and magnetic properties of the superlattice by chemical pressure or epitaxial strain.

\section{Methods}

Calculation of structure and ferroelectric polarization. Firstprinciples calculations are performed on $\mathrm{La}_{2} \mathrm{NiMnO}_{6}$ systems and on $\mathrm{R}_{2} \mathrm{NiMnO}_{6} / \mathrm{La}_{2} \mathrm{NiMnO}_{6}$ superlattices by using the Vienna $a b$ initio Simulation Package (VASP) within the framework of the projected augmented wave method ${ }^{34}$. As consistent with ref. 14, a specific chemical ordering is assumed for all these compounds so that, within the $\mathrm{B}$-sublattice, $\mathrm{Ni}$ atoms only have $\mathrm{Mn}$ atoms as first-nearest neighbours and vice versa. Practically, we investigate $\mathrm{R}=\mathrm{Ce}, \mathrm{Pr}, \mathrm{Nd}, \mathrm{Sm}, \mathrm{Gd}, \mathrm{Tb}, \mathrm{Dy}, \mathrm{Ho}$ and $\mathrm{Er}$, and $\mathrm{R}_{2} \mathrm{NiMnO}_{6} /$ $\mathrm{La}_{2} \mathrm{NiMnO}_{6}$ superlattices are built from $\mathrm{La}_{2} \mathrm{NiMnO}_{6}$ by replacing every other (001) LaO plane by a plane with $\mathrm{RO}$ composition (see Fig. 1 drawn by VESTA code ${ }^{35}$ ). The generalized gradient approximation GGA $+U$ method with the parameterization of Perdew-Burke-Ernzerh revised for solids (PBEsol) ${ }^{36}$ is used in the present work. The effective on-site Coulomb interaction is selected to be $3 \mathrm{eV}$ for both $\mathrm{Ni}^{2}+$ and $\mathrm{Mn}^{4+}$ ions, in line with typical choices in the literature ${ }^{19,37}$. We treat the valence electron configuration as follows: $5 s^{2} 5 p^{6} 5 d^{1} 6 s^{2}$ for La, Ce, Pr, Nd, Sm; $5 p^{6} 5 d^{1} 6 s^{2}$ for Gd, Tb, Dy, Ho, Er; $3 d^{9} 4 s^{1}$ for Ni; $3 d^{6} 4 s^{1}$ for Mn; and $2 s^{2} 2 p^{4}$ for $\mathrm{O}$. The $4 f$ electrons of rare-earth ions are thus kept as core electrons owing to the fact that partially filled $f$ orbitals are usually not well described by the current density functional theory (see, for example, ref. 34) and the properties studied in this article can be well mimicked without explicitly treating the $4 f$ electrons. For instance, Supplementary Fig. 2 shows that the experimental Curie temperatures of bulks $\mathrm{R}_{2} \mathrm{NiMnO}_{6}$ of ref. 14 can be very well reproduced without the need to incorporate $4 f$ electrons in the valence, which is consistent with the known facts that $4 f$ electrons only contribute to magnetism at very low temperature ${ }^{26}$. Similarly, we numerically found that the inclusion of the $4 f$ electrons has a relatively small effect on structural properties at $0 \mathrm{~K}$, as demonstrated by the change of the $\mathrm{a}, \mathrm{b}$ and $\mathrm{c}$ lattice parameters of $\mathrm{Gd}_{2} \mathrm{NiMnO}_{6}$ by only $0.5 \%, 0.08 \%$ and $0.4 \%$, respectively, with respect to the case when these $4 f$ electrons are kept in the core. We also use a plane wave energy cut-off of $500 \mathrm{eV}$ and a $6 \times 6 \times 4 k$-point mesh for Brillouin zone integrations. Structural optimizations were stopped when forces were $<0.005 \mathrm{eV}^{-1}$. The space group of the different phases encountered in the present work is determined by using the FINDSYM software ${ }^{38}$ and the electric polarization is calculated by the Berry phase approach ${ }^{39}$ (note that the electric polarization is evaluated by choosing the $\mathrm{P} 2_{1} / n$ ground state of bulk $\mathrm{La}_{2} \mathrm{NiMnO}_{6}$ as reference).

Calculation of magnetic Curie temperature. The in-plane and out-of-plane magnetic exchange constants $J_{\mathrm{Ni}-\mathrm{Mn}}$ determined from our first-principles calculations are taken as input for an effective Heisenberg Hamiltonian $H=1 / 2 \Sigma J_{\mathrm{Ni}-\mathrm{Mn}} \mathbf{S}_{\mathrm{Ni}} \cdot \mathbf{S}_{\mathrm{Mn}}$, where we assume classical spins and take $\left|\mathbf{S}_{\mathrm{Ni}}\right|=\left|\mathbf{S}_{\mathrm{Mn}}\right|=1$ for simplicity (these exchange constants are reported in Supplementary Tables 1 and 2). To compute the magnetic ordering temperatures, we solve such Hamiltonians by means of Monte Carlo simulations ${ }^{40}$ of a $20 \times 20 \times 20$ periodically repeated simulation supercell that contains 4,000 Ni spins and 4,000 Mn spins. We use 40,000 Monte Carlo sweeps for thermalization and 60,000 additional sweeps for computing statistical averages. Finally, note that we multiplied the first-principles-derived exchange constants of all the spin Hamiltonians used in this work by a factor of 0.685 , which is the correction needed so that the Heisenberg Hamiltonians reproduce all the experimental paramagnetic-toferromagnetic transition temperatures of the different $\mathrm{R}_{2} \mathrm{NiMnO}_{6}$ systems reported in ref. 14 (see Supplementary Fig. 2).

\section{References}

1. Ramesh, R. \& Spaldin, N. A. Multiferroics: progress and prospects in thin films. Nat. Mater. 6, 21-29 (2007).

2. Zanolli, Z., Wojdeł, J. C., Íñiguez, J. \& Ghosez, P. Electric control of the magnetization in $\mathrm{BiFeO}_{3} / \mathrm{LaFeO}_{3}$ superlattices. Phys. Rev. B 88, 060102 (2013).

3. Lee, J. H. et al. A strong ferroelectric ferromagnet created by means of spinlattice coupling. Nature 466, 954-958 (2010).

4. Ležaić, M. \& Spaldin, N. A. High-temperature multiferroicity and strong magnetocrystalline anisotropy in 3d-5d double perovskites. Phys. Rev. B 83, 024410 (2011).

5. Rocquefelte, X., Schwarz, K., Blaha, P., Kumar, S. \& van den Brink, J. Room-temperature spin-spiral multiferroicity in high-pressure cupric oxide. Nat. Commun. 4, 2511 (2013).

6. Mukherjee, S. et al. Room temperature nanoscale ferroelectricity in magnetoelectric $\mathrm{GaFeO}_{3}$ epitaxial thin films. Phys. Rev. Lett. 111, 087601 (2013).

7. Wang, W. et al. Room-temperature multiferroic hexagonal $\mathrm{LuFeO}_{3}$ Films. Phys. Rev. Lett. 110, 237601 (2013).

8. Eerenstein, W., Mathur, N. D. \& Scott, J. F. Multiferroic and magnetoelectric materials. Nature 442, 759-765 (2006).

9. He, Q. et al. Electrically controllable spontaneous magnetism in nanoscale mixed phase multiferroics. Nat. Commun. 2, 225 (2011).

10. Evans, D. M. et al. Magnetic switching of ferroelectric domains at room temperature in multiferroic PZTFT. Nat. Commun. 4, 1534 (2013).

11. Albrecht, D. et al. Ferromagnetism in multiferroic $\mathrm{BiFeO}_{3}$ films: a firstprinciples-based study. Phys. Rev. B 81, 140401(R) (2010).

12. Scott, J. F. Room-temperature multiferroic magnetoelectrics. NPG Asia Mater. 5, e72 (2013).

13. Azuma, M. et al. Designed Ferromagnetic, Ferroelectric $\mathrm{Bi}_{2} \mathrm{NiMnO}_{6}$. J. Am Chem. Soc. 127, 8889-8892 (2005).

14. Bull, C. L. \& McMillan, P. F. Raman scattering study and electrical properties characterization of elpasolite perovskites $L n_{2}\left(B B^{\prime}\right) \mathrm{O}_{6}(L n=\mathrm{La}, \mathrm{Sm} \ldots \mathrm{Gd}$ and $B$, $\left.B^{\prime}=\mathrm{Ni}, \mathrm{Co}, \mathrm{Mn}\right)$. J. Solid State Chem. 177, 2323-2328 (2004).

15. Bellaiche, L. \& Íñiguez, J. Universal collaborative couplings between oxygenoctahedral rotations and antiferroelectric distortions in perovskites. Phys. Rev. B 88, 014104 (2013).

16. Glazer, A. The classification of tilted octahedra in perovskites. Acta Crystallogr. Sect. B 28, 3384-3392 (1972).

17. Rondinelli, J. M. \& Fennie, C. J. Octahedral rotation-induced ferroelectricity in cation ordered perovskites. Adv. Mater. 24, 1961-1968 (2012).

18. Rogado, N. S., Li, J., Sleight, A. W. \& Subramanian, M. A. Magnetocapacitance and magnetoresistance near room temperature in a ferromagnetic semiconductor: $\mathrm{La}_{2} \mathrm{NiMnO}_{6}$. Adv. Mater. 17, 2225-2227 (2005).

19. Das, H., Waghmare, U. V., Saha-Dasgupta, T. \& Sarma, D. D. Electronic structure, phonons, and dielectric anomaly in ferromagnetic insulating double pervoskite $\mathrm{La}_{2} \mathrm{NiMnO}_{6}$. Phys. Rev. Lett. 100, 186402 (2008).

20. Zhao, H. J., Ren, W., Chen, X. M. \& Bellaiche, L. Effect of chemical pressure, misfit strain and hydrostatic pressure on structural and magnetic behaviors of rare-earth orthochromates. J. Phys. Condens. Matter 25, 385604 (2013).

21. Zhao, H. J., Ren, W., Yang, Y., Chen, X. M. \& Bellaiche, L. Effect of chemical and hydrostatic pressures on structural and magnetic properties of rare-earth orthoferrites: a first-principles study. J. Phys. Condens. Matter 25, 466002 (2013).

22. Shannon, R. Revised effective ionic radii and systematic studies of interatomic distances in halides and chalcogenides. Acta Crystallogr. Sect. A 32, 751-767 (1976).

23. Mulder, A. T., Benedek, N. A., Rondinelli, J. M. \& Fennie, C. J. Turning $\mathrm{ABO}_{3}$ antiferroelectrics into ferroelectrics: design rules for practical rotation-driven 
ferroelectricity in double perovskites and $\mathrm{A}_{3} \mathrm{~B}_{2} \mathrm{O}_{7}$ Ruddlesden-Popper compounds. Adv. Funct. Mater. 23, 4810-4820 (2013).

24. Kornev, I. A., Bellaiche, L., Janolin, P. E., Dkhil, B. \& Suard, E. Phase diagram of $\mathrm{Pb}(\mathrm{Zr}, \mathrm{Ti}) \mathrm{O}_{3}$ solid solutions from first principles. Phys. Rev. Lett. 97, 157601 (2006).

25. Yang, Y., Íñiguez, J., Mao, A.-J. \& Bellaiche, L. Prediction of a novel magnetoelectric switching mechanism in multiferroics. Phys. Rev. Lett. 112, 057202 (2014)

26. Booth, R. J. et al. An investigation of structural, magnetic and dielectric properties of $\mathrm{R}_{2} \mathrm{NiMnO}_{6}(\mathrm{R}=$ rare earth, Y). Mater. Res. Bull. 44, 1559-1564 (2009).

27. Pertsev, N. A., Zembilgotov, A. G. \& Tagantsev, A. K. Effect of mechanical boundary conditions on phase diagrams of epitaxial ferroelectric thin films. Phys. Rev. Lett. 80, 1988-1991 (1998).

28. Diéguez, O. et al. Ab initio study of the phase diagram of epitaxial $\mathrm{BaTiO}_{3}$. Phys. Rev. B 69, 212101 (2004).

29. Lai, B.-K., Kornev, I. A., Bellaiche, L. \& Salamo, G. J. Phase diagrams of epitaxial $\mathrm{BaTiO}_{3}$ ultrathin films from first principles. Appl. Phys. Lett. 86, 132904 (2005)

30. Guo, H. et al. Growth of epitaxial thin films of the ordered double perovskite $\mathrm{La}_{2} \mathrm{NiMnO}_{6}$ on different substrates. Appl. Phys. Lett. 89, 022509 (2006).

31. King, G. et al. Magnetic structures of $\mathrm{NaLMnWO}_{6}$ perovskites $(\mathrm{L}=\mathrm{Na}$, $\mathrm{Nd}$ and Tb). Phys. Rev. B 79, 224428 (2009).

32. Padhan, P. et al. Magnetodielectric effect in $\mathrm{Bi}_{2} \mathrm{NiMnO}_{6}-\mathrm{La}_{2} \mathrm{NiMnO}_{6}$ superlattices. J. Phys. Condens. Matter 21, 306004 (2009).

33. Scott, J. F. Data storage: multiferroic memories. Nat. Mater. 6, 256-257 (2007).

34. Kresse, G. \& Joubert, D. From ultrasoft pseudopotentials to the projector augmented-wave method. Phys. Rev. B 59, 1758-1775 (1999).

35. Momma, K. \& Izumi, F. VESTA 3 for three-dimensional visualization of crystal, volumetric andmorphology data. J. Appl. Cryst. 44, 1272-1276 (2011).

36. Perdew, J. P. et al. Restoring the density-gradient expansion for exchange in solids and surfaces. Phys. Rev. Lett. 100, 136406 (2008).

37. Zhu, M. et al. Electronic and magnetic properties of $\mathrm{La}_{2} \mathrm{NiMnO}_{6}$ and $\mathrm{La}_{2} \mathrm{CoMnO}_{6}$ with cationic ordering. Appl. Phys. Lett. 100, 062406 (2012).

38. Stokes, H. T. \& Hatch, D. M. FINDSYM: program for identifying the spacegroup symmetry of a crystal. J. Appl. Crystallogr. 38, 237-238 (2005).
39. King-Smith, R. D. \& Vanderbilt, D. Theory of polarization of crystalline solids. Phys. Rev. B 47, 1651-1654 (1993).

40. Escorihuela-Sayalero, C., Diéguez, O. \& Íñiguez, J. Strain engineering magnetic frustration in perovskite oxide thin films. Phys. Rev. Lett. 109, 247202 (2012).

\section{Acknowledgements}

This work is supported by National Science Foundation of China under Grant Nos. 51332006 (X.M.C. and H.J.Z.), 11274270 (X.M.C.) and 11274222 (W.R.), Program for Innovative Research Team in University of Ministry of Education of China (IRT13037) Shanghai Shuguang Program (12SG34) and the Eastern Scholar Program at Shanghai Institutions of Higher Education, Shanghai Municipal Education Commission. We thank the financial support of ARO Grant W911NF-12-1-0085 (W.R. and L.B.) and of the Department of Energy, Office of Basic Energy Sciences, under contract ER-46612 (Y.Y and L.B.). J.Í. had financial support from MINECO-Spain (Grant Nos. MAT2010-18113 and CSD2007-00041). Some computations were also made possible thanks to the MRI grant 0722625, MRI-R2 grant 0959124, and grant 0918970 from NSF, Shanghai Supercomputer Center, and a Challenge grant from the Department of Defense.

\section{Author contributions}

H.J.Z. performed the first-principles calculations presented in this article with help from W.R. Y.Y. conducted the Monte Carlo simulations with the help of J.I. X.M.C. and L.B. supervised the research work. All authors contributed to the discussion of the results and preparation of the manuscript.

\section{Additional information}

Supplementary Information accompanies this paper at http://www.nature.com/ naturecommunications

Competing financial interests: The authors declare no competing financial interests.

Reprints and permission information is available online at http://npg.nature.com/ reprintsandpermissions/

How to cite this article: Zhao, H. J. et al. Near room-temperature multiferroic materials with tunable ferromagnetic and electrical properties. Nat. Commun. 5:4021 doi: 10.1038/ncomms5021 (2014) 